\title{
Conditions for fixing violations of traffic rules do not pass pedestrians on unregulated pedestrian crossings in automatic mode
}

\author{
Samir R. Ibadov ${ }^{1}$, Boris Y. Kalmykov ${ }^{1}$, and Ragim R. Ibadov ${ }^{2, *}$ \\ ${ }^{1}$ Institute of Service and Entrepreneurship, Don State Technical University, 346500 Shakhty, Russia \\ ${ }^{2}$ Institute of Radio Engineering Systems and Control, Southern Federal University, Taganrog, Russia
}

\begin{abstract}
The article presents the urgency of developing systems for fixing violations of traffic rules in automatic mode. Describes the verification of conditions for determining the violation of pedestrians' nonadmission on unregulated pedestrian crossings by drivers of vehicles.
\end{abstract}

\section{The relevance of research}

The main type of traffic accidents (accidents) in Russia is a pedestrian impact, with over three quarters of these accidents associated with violations of the Traffic Regulations (SDA) by drivers of vehicles.

A total of 4,536 road accidents were registered in the Rostov Region in 2017 [1], in which 5,838 people were injured and 560 people died figure 1 .

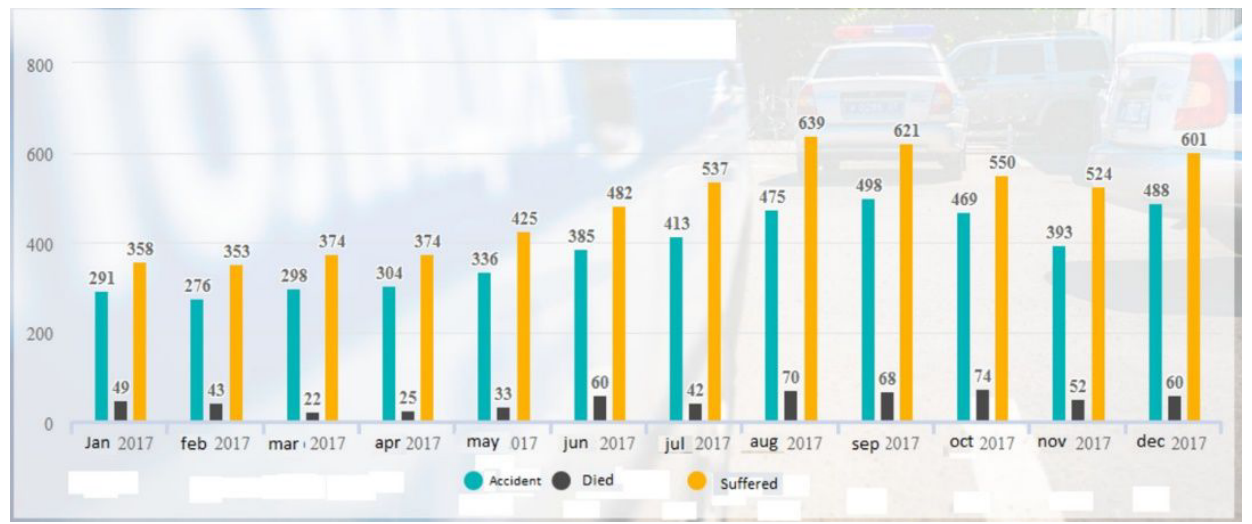

Fig.1. Accident in the entire Rostov region for 2017.

Of this number, 1148 traffic accidents were committed with a pedestrian collision [1], in which 1087 people were injured and 192 died, figure 2.

*Corresponding author: ragim_ibadov@mail.ru 


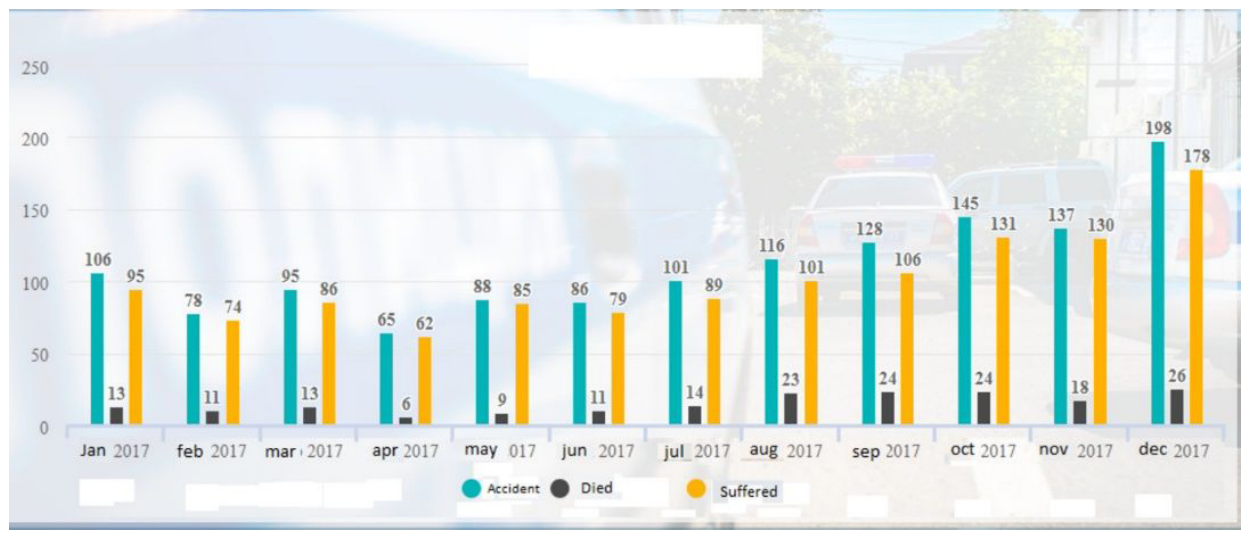

Fig.2. Accident with the impact on pedestrians in the Rostov region in 2017

On the regulated pedestrian crossings, 148 road accidents were recorded [1], 149 people were injured, 7 people were killed in 2017, figure 3 .

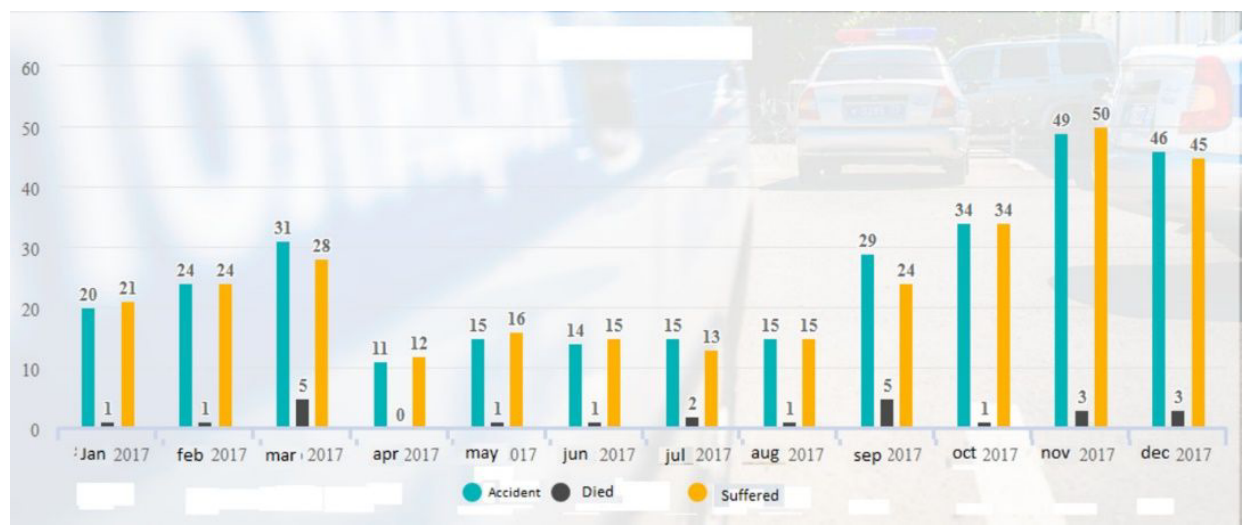

Fig. 3. Accident at unregulated pedestrian crossings for the Rostov region in 2017

Traffic accidents at unregulated pedestrian crossings [1], 303 injured 297 people, 24 died, figure 4.

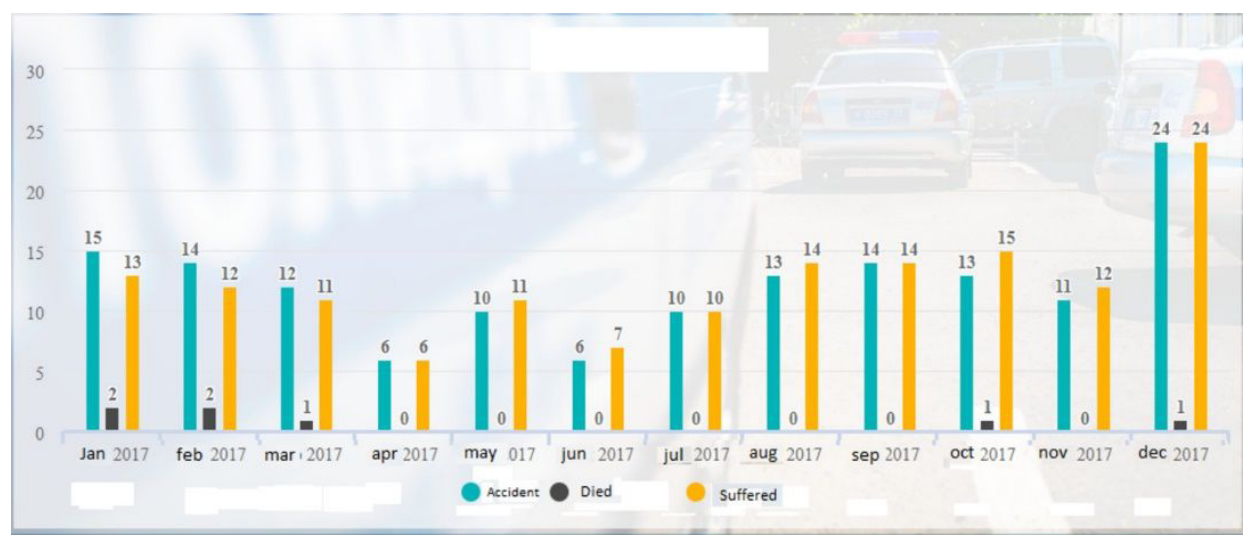

Fig.4. Accident on adjustable pedestrian crossings for the Rostov region in 2017. 
As can be seen from the statics, every fourth accident, from the number registered for the Rostov region, occurs with a collision on pedestrians. At the same time, twice as many road accidents occurred on unregulated pedestrian crossings, and twice as many people were wounded in comparison with regulated pedestrian crossings.

In this regard, the urgent task is the development and implementation of photo-video systems to fix violations of the Rules of the Road on regulated and unregulated pedestrian crossings. As practice shows, photo-video systems fixing violations cause drivers to be more vigilant on the roads and comply with high-speed modes, and the choice of wrong speed is often the result of an accident.

\section{Conditions for determining the violation}

In order to determine whether the driver should miss a pedestrian on the pedestrian crossing and if he should, to establish whether he did it or not, it is necessary to check a number of conditions. Whether there was an opportunity to brake at the driver when the pedestrian has approached to edge of a pedestrian crossing. In the textbooks [1, 2], safe speeds are considered according to the simplified scheme, depending on the distance $\mathrm{y}_{\Pi}$, on which the pedestrian was from the traffic area of the vehicle for a given distance $S_{y}$, pedestrian speed $\mathrm{V}_{\Pi}$ and the level of limiting deceleration $j_{T}$. The pedestrian is conventionally taken as a point, and the dimensions of the vehicle are of length $L$ and width $B$. The trajectories of the vehicle and pedestrian traffic intersect at an angle of 90 degrees.

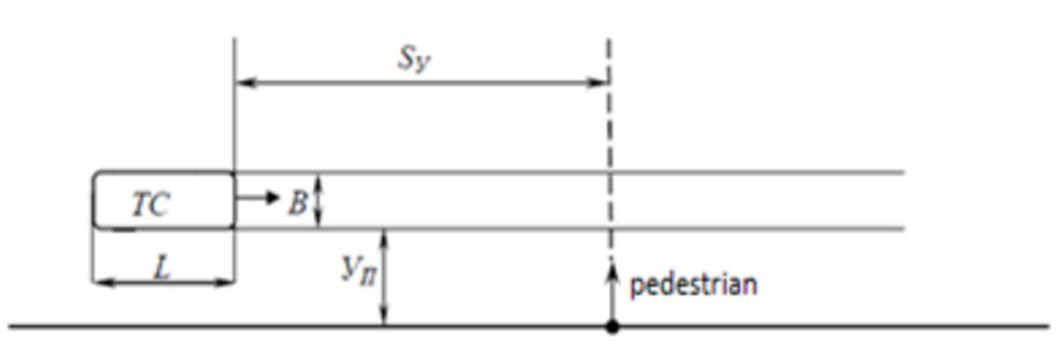

Fig.5. Scheme for calculating safe speeds.

V1 corresponds to the condition of stopping the car by emergency braking to the point of arrival (pedestrian traffic line):

$$
V_{1}=T \cdot j_{T}\left(\sqrt{2 S_{y} / T^{2} \cdot j_{T}+1}-1\right)
$$

V3 speed at which the pedestrian has time to leave the lane of the car before approaching the latter:

$$
V_{3}=S_{y} \cdot V_{\Pi} /\left(Y_{\Pi}+B\right)
$$

V4 speed at which the car manages to pass a pedestrian, applying emergency braking:

$$
V_{4}=\frac{2 S_{y}+\left(t_{n}-T\right)^{2} \cdot j T}{2 \cdot t_{n}}=V_{3}+\left(t_{\Pi}-T^{2}\right) \cdot j T / 2 t_{\Pi} .
$$

If it was possible, the driver must pass a pedestrian, if the driver did not do it and drove, then the pedestrian started crossing the road on a pedestrian crossing, this would be considered a violation. Also, by letting pedestrians from one side of the road, the driver 
should make sure that pedestrians do not go from the opposite side of the road and only then continue driving. Consider this schematically for a single-lane road. The car, moving on the right lane of traffic, approaching an unregulated pedestrian crossing, is obliged to pass the pedestrian standing at the edge of the pedestrian crossing figure 6 .

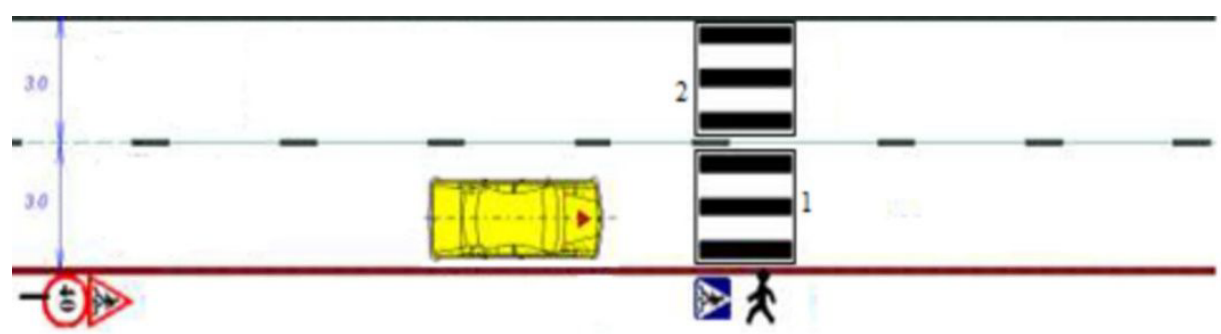

Fig. 6. The layout of the pedestrian and the car on an unregulated pedestrian crossing.

After the pedestrian passes from one lane marked by number 1 to another lane marked with number 2 , the driver making sure that there are no pedestrians from lane 2 on lane 1 can continue to move along lane 1 figure 7 .

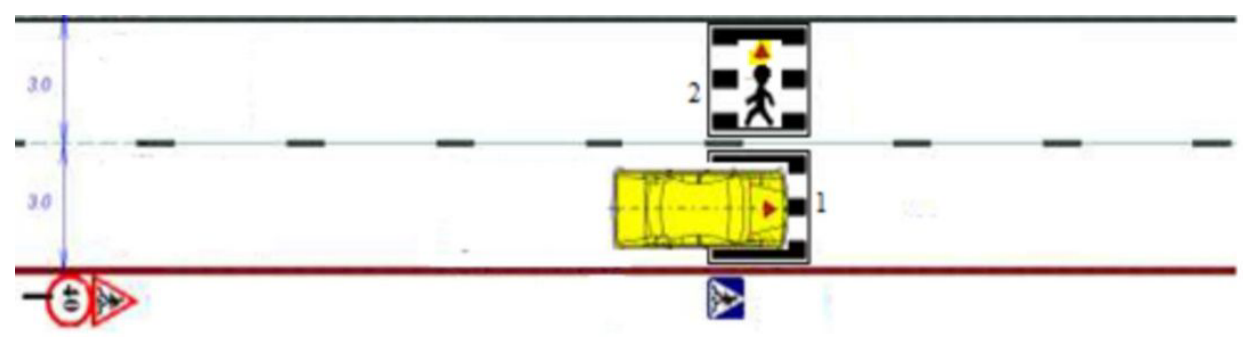

Fig. 7. The scheme of the location of the pedestrian and the car at the moment at the unregulated pedestrian crossing at the time.

After the pedestrian passes from one lane marked by number 1 to another lane marked with number 2 , the driver making sure that there are no pedestrians from lane 2 on lane 1 can continue to move along lane 1 figure 7.

\section{References}

1. B. Ye. Borovsky, The traffic safety of the automobile transport (Lenizdat, Leningrad, 1984)

2. E.R. Domke, Investigation and examination of road transport Incidents: a textbook for students of higher educational institutions (Publishing Center "Academy", Moscow, 2009)

3. S. Ibadov, R. Ibadov, B. Kalmukov, V. Krutov, MATEC Web Conf, 132, 05005 (2017) 